\title{
Pretreatment with the monoacylglycerol lipase inhibitor URB602 protects from the long-term consequences of neonatal hypoxic-ischemic brain injury in rats
}

\author{
Silvia Carloni ${ }^{1}$, Daniel Alonso-Alconada ${ }^{2}$, Silvia Girelli ${ }^{1}$, Andrea Duranti ${ }^{3}$, Andrea Tontini ${ }^{3}$, Daniele Piomelli ${ }^{4,5}$, Enrique Hilario ${ }^{2}$, \\ Antonia Alvarez ${ }^{2}$ and Walter Balduini ${ }^{1}$
}

BACKGROUND: The endocannabinoids are emerging as natural brain protective substances that exert potentially beneficial effects in several neurological disorders by virtue of their hypothermic, immunomodulatory, vascular, antioxidant, and antiapoptotic actions. This study was undertaken to assess whether preventing the deactivation of the endocannabinoid 2-arachidonoylglycerol (2-AG) with the monoacylglycerol lipase (MAGL) inhibitor URB602 can provide neuroprotective effects in hypoxia-ischemia (HI)-induced brain injury.

METHODS: URB602 was administered into the right lateral ventricle 30 min before 7 -day-old pup rats were subjected to $\mathrm{HI}$. The neuroprotective effect was evaluated on postnatal day (PN) 14 or at adulthood (PN80) using behavioral and histological analyses. Activated caspase-3 expression and propidium iodide labeling were assessed as indexes of apoptotic and necrotic cell death, respectively.

RESULTS: Pretreatment with URB602 reduced apoptotic and necrotic cell death, as well as the infarct volume measured at PN14. At adulthood, URB602-treated HI animals performed better at the T-maze and the Morris maze, and also showed a significant reduction of brain damage.

CONCLUSION: These results demonstrate that a pretreatment with URB602 significantly reduces brain damage and improves functional outcome, indicating that endocannabinoiddegrading enzymes may represent an important target for neuroprotection in neonatal ischemic brain injury.

$\mathrm{n}$ the past decades, important advances have been made in cannabinoid $(\mathrm{CB})$ research, with the endocannabinoids emerging as natural brain protective substances. These are part of a new intercellular communication network, called the endogenous $\mathrm{CB}$ system (1). The endocannabinoid system comprises the $\mathrm{CB}$ receptors ( $\mathrm{CB} 1, \mathrm{CB} 2$, and non- $\mathrm{CB} 1 / \mathrm{CB} 2$ receptors), the endocannabinoids $\mathrm{N}$-arachidonylethanolamine (anandamide; AEA), 2 -arachidonylglycerol (2-AG), and the enzymes responsible for endocannabinoid biosynthesis, transport, and degradation (1).
Acting as retrograde messengers, endocannabinoids provide neuromodulatory functions in the brain, regulating processes such as motor activity, memory and learning, appetite, emesis, nociception, and the survival or death decision of neural cells after harmful insults $(1,2)$. They exert potentially beneficial effects by virtue of their hypothermic, immunomodulatory, vascular, antioxidant, and antiapoptotic actions $(3,4)$, which can generate a neuroprotective response after several neurological disorders.

Following an ischemic brain injury, there is an increased production and accumulation of endocannabinoids in the brain $(5,6)$, and upregulation of CB receptors $(7,8)$. Activation of the endocannabinoid system has been extensively evaluated as a possible target for the treatment of cerebral ischemia $(7,9,10)$, trauma (11), and excitotoxicity $(12,13)$. Enhanced levels of endocannabinoids have also been observed in the brain after an acute excitotoxic insult in newborn rats and exogenous administration of synthetic or endocannabinoids has shown neuroprotective effects both in vitro and in vivo $(7,14,15)$.

After activation of $\mathrm{CB}$ receptors, endocannabinoids are rapidly inactivated by reuptake and enzymatic hydrolysis, therefore limiting their potential protective effects. Termination of endocannabinoid signaling is carried out primarily by fatty acid amide hydrolase, which degrades AEA, and monoacylglycerol lipase (MAGL), which hydrolyzes 2-AG (1). Because the effects of exogenous CB receptor agonists do not appear too specific (16), currently interest is gradually shifting to the alternative approach of amplifying the effects of endocannabinoids by preventing their deactivation. Indeed, an interesting aspect of endocannabinoid activity is the rapid induction of their synthesis, receptor activation, and degradation. On these bases, the endocannabinoid system has been suggested to act on demand, with a tightly regulated spatial and temporal selectivity (1). The system, therefore, exerts its modulatory actions only when and where it is needed and this fact poses an important distinction between the physiological functions of the

\footnotetext{
The first two authors contributed equally to this work.

'Department of Biomolecular Sciences, Section of Pharmacology and Pharmacognosy, University of Urbino "Carlo Bo," Urbino (PU), Italy; ${ }^{2}$ Department of Cell Biology and Histology, School of Medicine, University of the Basque Country, Leioa, Spain; ${ }^{3}$ Department of Biomolecular Sciences, Medicinal Chemistry and Technology Unit, University of Urbino "Carlo Bo," Urbino (PU), Italy; ${ }^{4}$ Department of Pharmacology, University of California, Irvine, Irvine, California; ${ }^{5}$ Department D3, Italian Institute of Technology, Genoa, Italy. Correspondence:Walter Balduini (walter.balduini@uniurb.it)
} 
endocannabinoid system and the pharmacological actions of exogenous CB receptor agonists, which lack such selectivity. Thus, by using "endocannabinoid enhancers" instead of exogenous CBs, it may be possible to avoid ubiquitous receptor activation, allowing the activation of the endocannabinoid system in a temporally and spatially restricted manner. This approach has been recently seen in rat organotypic hippocampal slices where a protective effect of the MAGL inhibitor URB602 was reported (17).

This study was undertaken to evaluate the potential in vivo neuroprotective properties of URB602 in neonatal hypoxicischemic brain injury.

\section{RESULTS}

\section{URB602 Reduces the Brain Damage Induced by Neonatal HI}

To assess the effect of MAGL inhibition on brain damage and find the dose to be used in long-term experiments, different groups of animals were treated with intracerebroventricular injection $30 \mathrm{~min}$ before hypoxia-ischemia (HI) with several doses of URB602. In these experiments, animals were euthanized and evaluated for histological damage 7 days after HI. Figure 1 shows a quantitative evaluation of the effect of the different treatments on brain damage. These experiments revealed a significant reduction of brain injury when URB602 was administered at doses of $12.5 \mu \mathrm{g}$
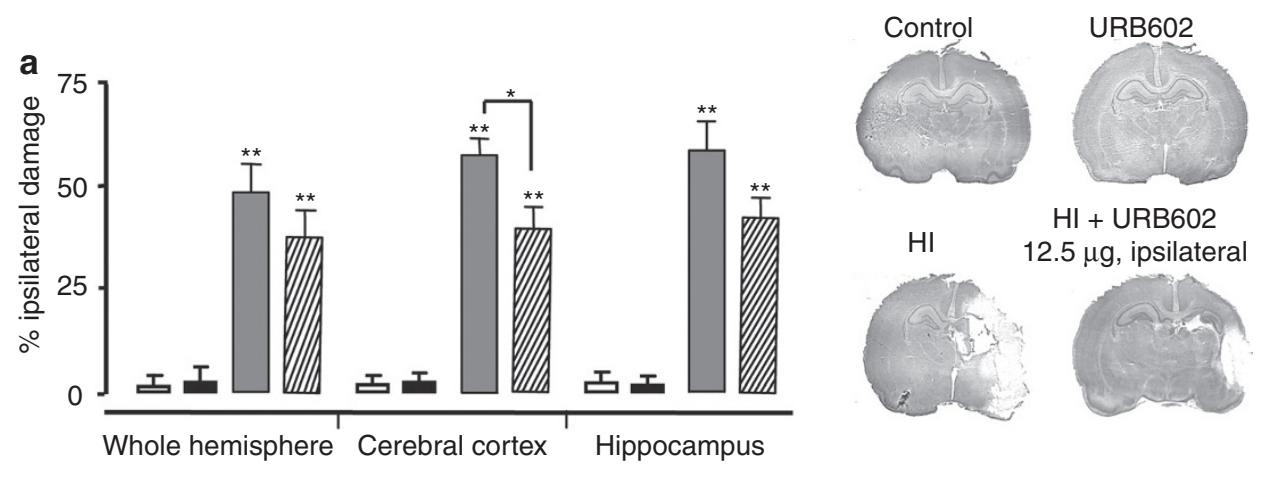

HI + URB602

Control

URB602
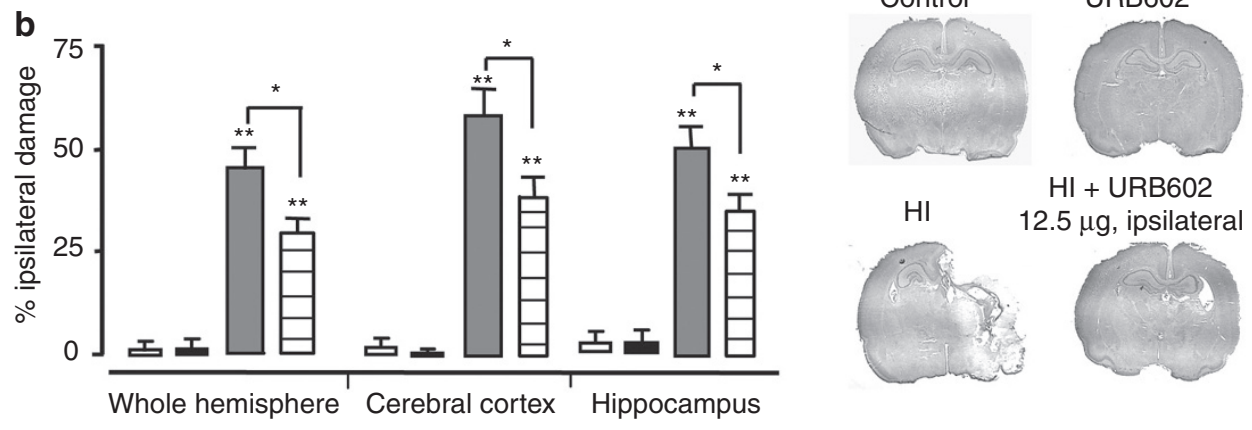

$\mathrm{HI}+\mathrm{URB} 602$

$\mathrm{HI}$

$12.5 \mu \mathrm{g}$, ipsilateral
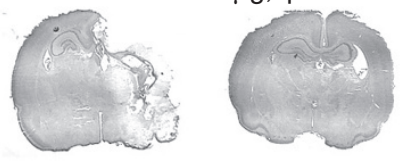

Control

URB602

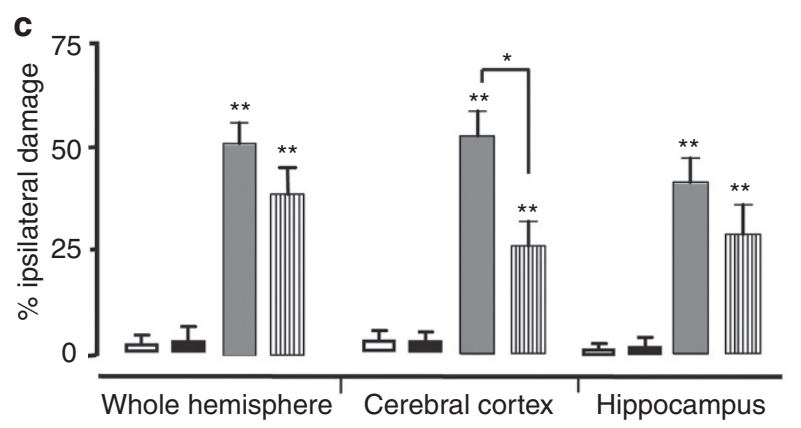

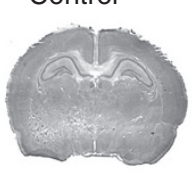

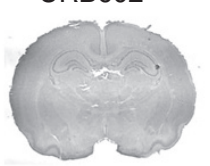

$\mathrm{HI}+\mathrm{URB} 602$

HI

$12.5 \mu \mathrm{g}$, ipsilateral
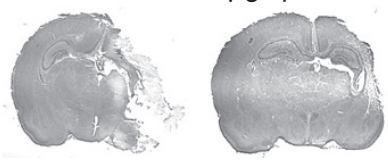

Figure 1. Effect of URB602 on neonatal hypoxic-ischemic brain injury. Infarct volume measured in 14-day-old rats subjected to hypoxia-ischemia (HI) on postnatal day 7 and treated with vehicle (gray columns, HI) or with URB602 (hatched columns, $\mathrm{HI}+\mathrm{URB} 602$ ) at a dose of (a) $12.5 \mu \mathrm{g}$ or (b) $25 \mu \mathrm{g}$ in the ventricle ipsilateral to the occluded carotid, or at a dose of (c) $50 \mu \mathrm{g}$ administered in the ventricle contralateral to the occluded carotid. The control groups received a corresponding volume of vehicle (white columns, control) or URB602 (black columns, URB602). Right side of the figure: representative photomicrographs of each experimental group. Results are expressed as percentage of ipsilateral damage. ${ }^{* *} P<0.001$ as compared with the control groups; ${ }^{*} P<0.05$ comparing the percentage of ipsilateral damage of ischemic animals with that of URB602-treated ischemic animals; one-way ANOVA was followed by Newman-Keuls post hoc test. Results are shown as mean \pm SEM ( $n=8 /$ group, $\mathbf{a} ; n=10 /$ group, $\mathbf{b}$ and $\mathbf{c}$ ). Volumes for the whole hemisphere of the experimental groups treated with $25 \mu \mathrm{g}$ of URB602 were $\left(\mathrm{mm}^{3}\right.$; L, contralateral; R, ipsilateral): control, L $324 \pm 12.6, \mathrm{R} 323.5 \pm 33.1$; URB602, $\mathrm{L} 357.2 \pm 8.9, \mathrm{R} 354.6 \pm 43.4 ; \mathrm{HI}, \mathrm{L} 293.9 \pm 29.04, \mathrm{R} 164.9 \pm 41.6 ; \mathrm{HI}+\mathrm{URB} 60225 \mu \mathrm{g}, \mathrm{L} 364.3 \pm 12.4, \mathrm{R} 224.3 \pm 16.31 ; P<0.05$ comparing the volume of right hemisphere of ischemic animals with that of $25 \mu \mathrm{g}$ URB602-treated ischemic animals, Mann-Whitney $\mathrm{U}$ test. 


\section{Articles | Carlonietal.}

and $25 \mu \mathrm{g}$ (Figure 1a,b) into the right ventricle, ipsilateral to the occluded carotid, or when a dose of $50 \mu \mathrm{g}$ was injected into the left ventricle, contralateral to the occluded carotid (Figure 1c). The percentage reduction of brain volume calculated for the whole hemisphere, the cerebral cortex and the hippocampus for vehicle-treated ischemic animals and ischemic animals treated with a dose of $25 \mu \mathrm{g}$ URB602 were 48.9 vs. $35.8 \%$, 61.8 vs. $42.2 \%$, and 52.2 vs. $40.3 \%$, respectively. These data indicate that administration of the MAGL inhibitor URB602 results in neuroprotection. The dose of $25 \mu \mathrm{g}$ per rat was selected for evaluating the neuroprotective effect of URB602 in short-term biochemical studies and long-term behavioral studies.

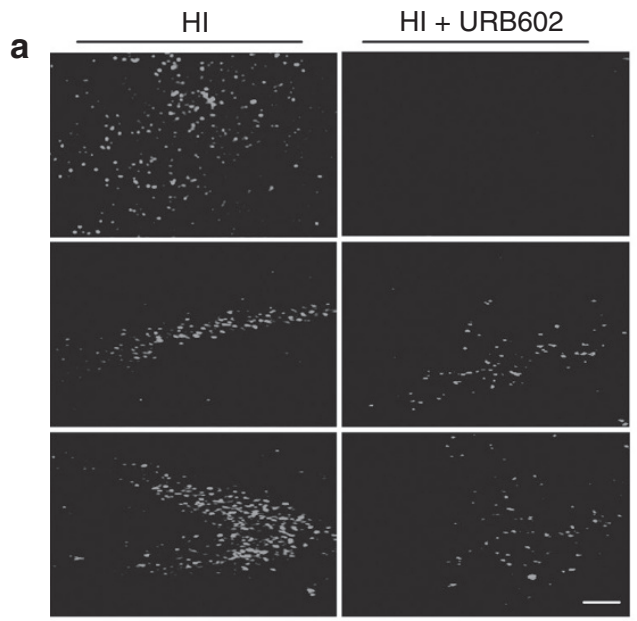

C

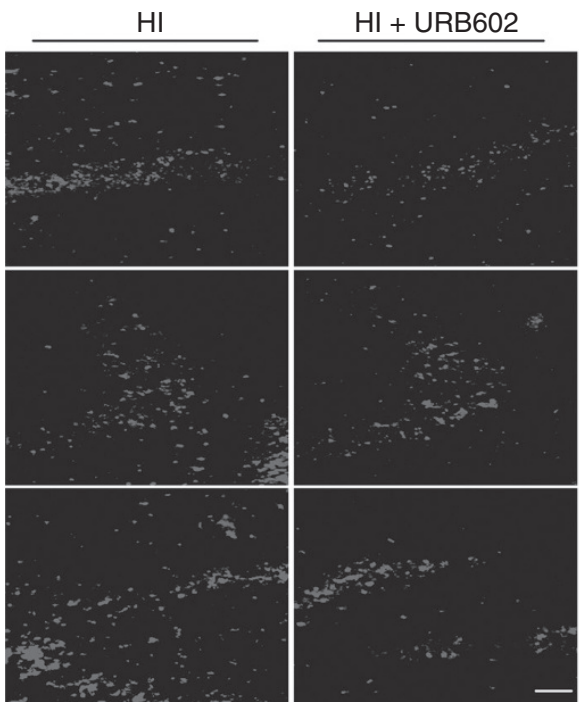

URB602 Reduces Caspase-3 Activation and Propidium lodide Labeling Induced by Neonatal HI

As shown in Figure 2a, after treatment with URB602 the number of cleaved caspase-3-positive cells appeared markedly reduced in the cerebral cortex (Figure 2a, top panels) and in the CA1 (Figure 2a, middle panels) and CA2/CA3 (Figure 2a, bottom panels) hippocampal regions. The reduction of caspase- 3 activation was confirmed by western blot experiments performed in the cortical tissue (Figure $2 \mathbf{b}$ ). As shown in Figure 2c, $24 \mathrm{~h}$ after HI, the number of cells positive to propidium iodide, a fluorescent dye used in vivo to stain necrotic cells (18), was significantly reduced after URB602 treatment. Taken together, these results indicate
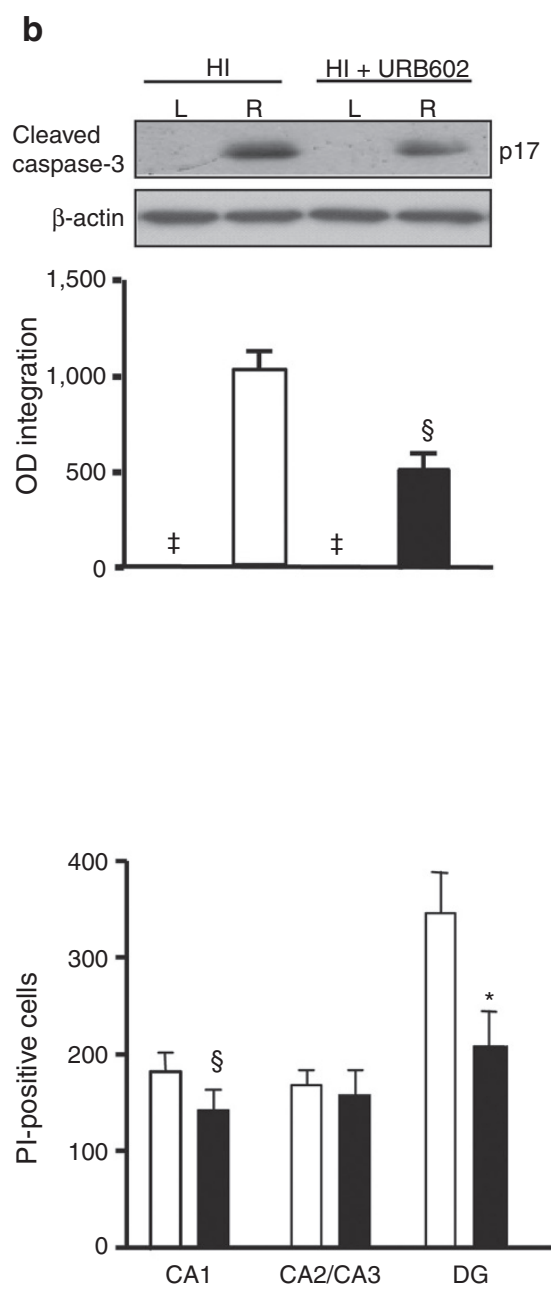

Figure 2. Effects of neonatal hypoxia-ischemia (HI) and of treatment with URB602 on apoptotic and necrotic cell death. (a) Representative photomicrographs showing the expression of cleaved caspase- 3 in the lesioned side of the cerebral cortex (top panels) and hippocampus (CA1 (middle panels) and CA2/CA3 (bottom panels)) of ischemic (HI) and URB602-treated ischemic animals (HI + URB602). (b) Representative western blots and quantitative evaluation of cleaved caspase-3 (p17) expression in ischemic (HI) and URB602-treated ischemic animals (HI + URB602) 24h after HI. Data are expressed as optical density (OD) integration and are the mean \pm SEM ( $n=5 /$ group). L, left side, contralateral; $R$, ipsilateral to occluded carotid artery; $\neq$, nondetectable, $\S P<0.01$; one-way ANOVA was followed by Newman-Keuls multiple comparison test. $\beta$-actin was run as an internal standard. (c) Photomicrographs showing cells labeled with propidium iodide (PI) and PI-positive cells counted in the lesioned side of the three hippocampus regions (CA1 (top panels), CA2/CA3 (middle panels) and dentate gyrus (DG) (bottom panels)) of ischemic (HI) and URB602-treated ischemic animals (HI + URB602). Data are reported as the mean \pm SEM ( $n=5 /$ group). The green (caspase-3) and red (PI) colors were converted to grayscale. ${ }^{*} P<0.05$ and $\S P<0.01$ as compared with the HI group; Mann-Whitney U test. Scale bars $=100 \mu \mathrm{m}$. 
that the administration of URB602 reduces both apoptotic and necrotic cell death.

\section{URB602 Reduces HI-Induced Long-Lasting Behavioral Alterations and Brain Damage}

We next tested if URB602 administration could also improve the long-lasting behavioral alterations observed in this model of neonatal HI. URB602 treatment did not affect the animal growth rate, assessed as body weight at weaning (21d old: control 51.2 \pm 1.0 ; URB602 $53 \pm 2.4$; HI $49.3 \pm 0.9 ; \mathrm{HI}+\mathrm{URB} 602$ $54.8 \pm 1.1 \mathrm{~g} ; P>0.05$ ) and adulthood (80 d old: control 389.6 \pm 12; URB602 $393 \pm 7.9 ;$ HI $402.2 \pm 9.1 ; \mathrm{HI}+\mathrm{URB} 602409$ $\pm 6.3 \mathrm{~g} ; P>0.05)$. When tested in the T-maze from postnatal day (PN) 28 to PN30, in agreement with our earlier studies $(18-21)$, ischemic rats preferentially chose the arm ipsilateral to the damaged side $(P<0.01)$. The percentage of right/left
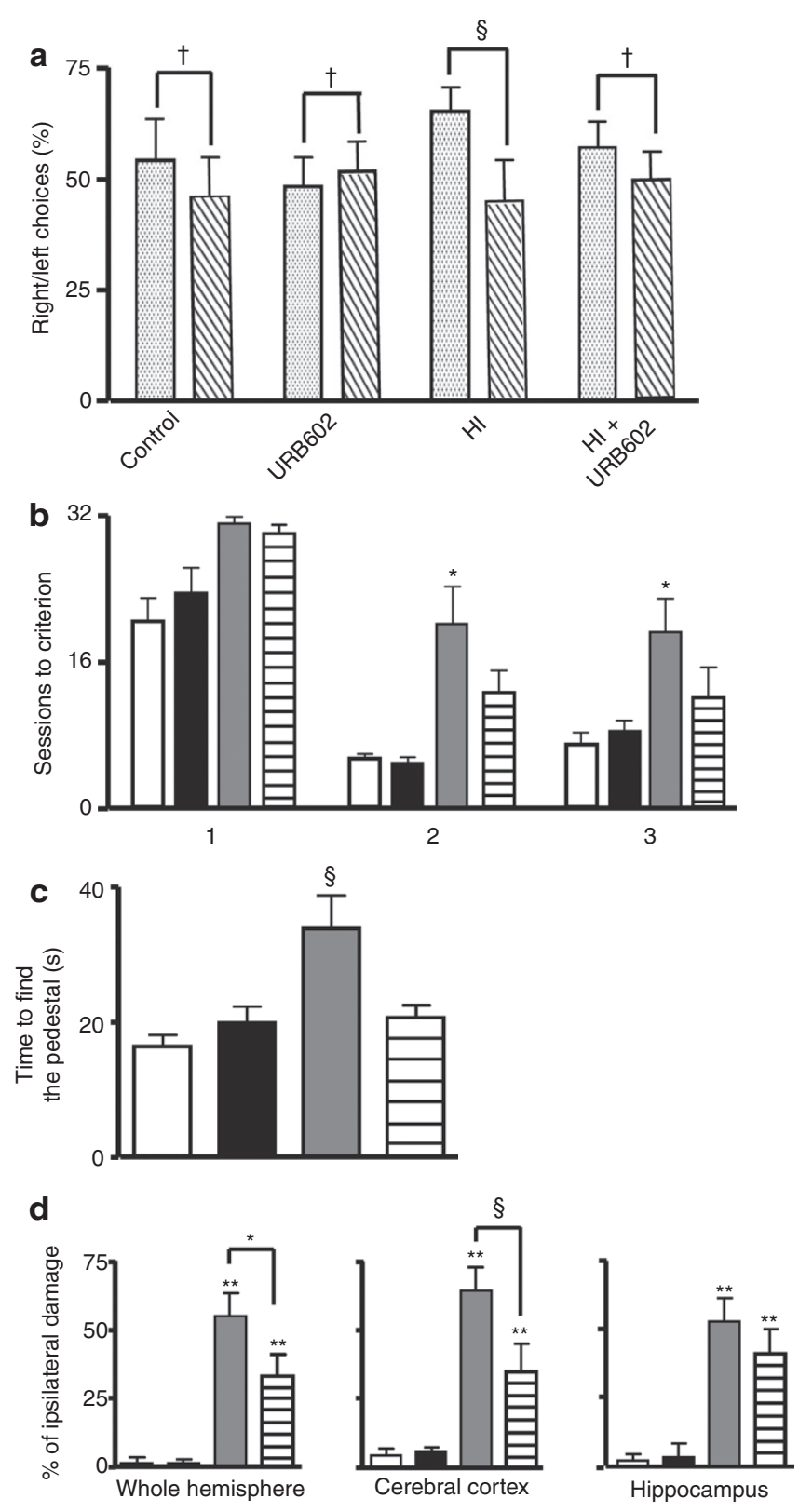

choices of HI + URB602 animals, although higher, was not statistically different (Figure 3a).

Learning abilities were tested in a Morris maze using a training-to-criterion test from PN45 to PN57 (Figure 3b). Groups did not differ in the number of sessions required to find the submerged pedestal in the first position $(P=0.056)$. However, the HI group had significantly impaired performances compared with controls when the pedestal was placed in the second or in the third position (Figure $3 \mathbf{b}, P<0.05$ ). The URB602-treated ischemic group took slightly more than half the number of sessions needed by the ischemic group to reach the criterion ( $11 \pm 2$ vs. $19 \pm 3$, respectively). After 15 days, (PN72) animals were tested for retention (Figure 3c). In this test, only ischemic rats differed significantly from controls in the time required to find the submerged pedestal $(P<0.01)$. The URB602-treated ischemic group took a shorter time to find the pedestal as compared with the ischemic group (20.5 \pm 3.6 vs. $33.5 \pm 5.2 \mathrm{~s}$, respectively). It should also be noted that URB602-treated control animals did not differ from the control group in both tests.

Animals were euthanized at the end of the behavioral tests (PN80) and evaluated for brain damage. A quantitative evaluation of brain damage at adulthood is reported in Figure 3d. No differences were observed between the whole-brain volume of control and URB602-treated animals. Adult ischemic animals, however, showed a marked degeneration of the hemisphere on the occluded side. No injury was evident on the left side of the forebrain, cerebellum, or brainstem. Brain damage was significantly reduced in URB602-treated ischemic animals (Figure 3d).

\section{DISCUSSION}

In this study we show that pretreatment with URB602 protects from brain damage in a neonatal model of HI. We also show that the neuroprotective effect of URB602 is long lasting, as both brain injury and behavioral outcomes were significantly improved when ischemic animals were assessed at adulthood.

Figure 3. Effects of neonatal hypoxia-ischemia $(\mathrm{HI})$ and of treatment with URB602 on T-maze, Morris water maze performances, and brain injury. Ischemic animals were treated with vehicle (gray columns, $\mathrm{HI}$ ) or with URB602 at the dose of $25 \mu \mathrm{g}$ (hatched columns, HI + URB602). The control groups received a corresponding volume of vehicle (white columns, control) or URB602 (black columns, URB602). (a) Spontaneous alternation in a T-maze. Results are expressed as percentage of right/left choices. $\dagger$, not significant, $\S P<0.01$; Mann-Whitney $U$ test. (b) Morris water maze performances. Data are expressed as the number of sessions needed to reach the criterion, that is, three trials in a row with an average escape of $<20 \mathrm{~s}$. Results for the first (1), second (2), and third (3) position of the pedestal are shown, ${ }^{*} P<0.05$ compared with the control group; Kruskal-Wallis test. (c) Retention. Animals were tested 15 days after the acquisition test (postnatal day 72). Data are expressed as the total time taken to find the pedestal, $\S P<0.01$, as compared with the control group; one-way ANOVA was followed by Newman-Keuls multiple comparison test. (d) Whole hemisphere and regional brain injury of ischemic and URB602-treated ischemic rats killed at the end of behavioral experiments. Results (mean \pm SEM) are expressed as percentage of ipsilateral damage. ${ }^{*} P<0.001$ as compared with the control groups; ${ }^{*} P<0.05$ and $\S P<0.01$ comparing the percentage of ipsilateral damage of ischemic animals with that of URB602treated ischemic animals; one-way ANOVA was followed by NewmanKeuls post hoc test. Results are shown as mean \pm SEM ( $n=10 /$ group). 
Indeed, URB602-treated animals did not differ from controls in either the T-maze or the water maze, and this cognitive improvement correlated with a significant reduction of brain damage in the cerebral cortex and hippocampus, brain areas known to be essential for acquisition and retention of spatial memory tasks. Biochemical evidence indicates that URB602induced reduction of brain injury was associated with decreased apoptotic pathway activation (reduced activation of caspase-3), and occurred without shifting to necrotic cell death (decreased number of propidium iodide-positive cells) (18).

A growing body of evidence indicates that CBs modulate pathways involved in numerous harmful events $(3,4)$ and show neuroprotective effects in different paradigms of hypoxicischemic brain injury $(7,14,22)$. It is well known that ischemic brain tissue produces and accumulates both 2-AG and AEA, whose protective role has been highlighted either by their exogenous administration or by the inhibition of their metabolic enzymes $(9,10,15)$. Thus, it is not surprising to consider endocannabinoids as endogenous protective molecules and, by extension, the endocannabinoid system as a potential target for new drugs that could improve ischemic injury. However, the effects of exogenous $\mathrm{CB}$ receptor agonists do not appear to be very specific (16), and currently the interest is gradually shifting to the alternative approach of amplifying the effects of endocannabinoids by preventing their deactivation. Thus, by using these "endocannabinoid enhancers" instead of exogenous CBs, it may be possible to avoid ubiquitous receptor activation. MAGL is the main enzyme involved in 2-AG hydrolysis (23), and specific MAGL inhibitors could block 2-AG deactivation and therefore amplify its intrinsic actions. URB602 is known to increase 2-AG levels and enhance 2-AG-mediated signaling in neurons $(24,25)$, without affecting the activities of other lipid-metabolizing enzymes such as fatty acid amide hydrolase (which hydrolyzes AEA), diacylglycerol lipase, or COX-2 $(25,26)$. On these bases, URB602 is considered to be a selective inhibitor of MAGL activity that may therefore regulate the 2-AG-CB1 receptor signaling. URB602 also possess anti-inflammatory effects (27) that have been correlated to the protective effects of exogenous 2-AG, which lessen inflammation via reduction of nuclear factor- $\mathrm{\kappa B}$ and COX-2 activation and cytokine and reactive oxygen species formation $(11,28,29)$.

This study shows that URB602 is neuroprotective in a neonatal model of HI, but the mechanism(s) by which neuroprotection was achieved was not investigated. Our results are in accordance with those recently reported by Landucci et al. (17) showing that URB602 had protective effects in a model of ischemia in rat organotypic hippocampal slices. They also found that the effect of URB602 was comparable with that observed after exogenous 2-AG administration and that neuroprotection was blocked by the CB1 antagonist AM251. Furthermore, they reported that blocking the fatty acid amide hydrolase/ AEA pathway with URB597 did not have protective effect, indicating that it is the amplification of the MAGL/2-AG pathway that might play a pivotal role in the neuroprotective effect of the endocannabinoid system (17). Accordingly, preliminary results obtained with the fatty acid amide hydrolase inhibitor
URB597 $(17,25)$ in our model of HI have shown that this compound does not have protective effects (data not shown). Taken together, this evidence makes us confident that the protective effect of URB602 observed after in vivo administration could be mediated by its effect on MAGL, although CB-independent effects of the compound cannot be excluded.

In conclusion, the results of this study indicate that treatment with URB602 is neuroprotective and has long-lasting beneficial effects on HI occurring during brain development. Even though further investigation is required to elucidate the underlying bases for its neuroprotective effect, the well-established effect of URB602 with the endocannabinoid degrading enzyme MAGL $(24,25)$ supports the hypothesis that the approach of amplifying the effects of endocannabinoids by preventing their deactivation can offer a promising therapeutic approach for reducing ischemia-induced neurodegeneration in the neonate. Before these agents can be proposed for treating asphyxiated fetus or newborns, however, further studies must determine if they are effective when systemically administered after the ischemic insult and if they can present toxicity in the newborn.

\section{METHODS}

\section{Animals}

All surgical and experimental procedures were carried out in accordance with the Italian regulations for the care and use of laboratory animals, and were approved by the animal care committee of the University of Urbino "Carlo Bo." Female Sprague-Dawley rats (Charles River, Milan, Italy) were housed with breeder males and conception determined by vaginal smear. Pregnant rats were then housed in individual cages and the day of delivery was considered day 0 for the pups. On PN1, litters of pups born on the same day were randomized and reduced to 10 male rats per litter. Each litter contained pups assigned to the different groups of treatment with the purpose of circumventing litter effects.

\section{Cerebral HI}

The procedure used was a slight modification of the one described by Rice et al. (30). Seven-day-old animals were anesthetized with $3 \%$ isoflurane in a $\mathrm{N}_{2} \mathrm{O} / \mathrm{O}_{2}(70 / 30 \%)$ mixture and the right common carotid artery of each pup was exposed, isolated from nerve and vein, and ligated with surgical silk. The wound was then sutured and the animal allowed to recover for $3 \mathrm{~h}$ under a heating lamp. Pups were then placed in airtight jars and exposed for $2.5 \mathrm{~h}$ to a humidified nitrogenoxygen mixture ( $92 \%$ and $8 \%$, respectively) delivered at $5-6 \mathrm{l} / \mathrm{min}$. The jars were partially submerged in a $37^{\circ} \mathrm{C}$ water bath to maintain a constant thermal environment. Rectal temperature was measured before surgery and at the end of the period of hypoxia. No differences were found among groups (data not shown).

\section{Drug Synthesis and Administration}

URB602 (biphenyl-3-ylcarbamic acid cyclohexyl ester; Figure 4) was synthesized as described earlier (31) or, alternatively, as follows. To a solution of biphenyl-3-yl isocyanate (1 equivalent), obtained from the corresponding carboxylic acid, in cyclohexanol, $\mathrm{CuX}(X$ $=\mathrm{Cl}, \mathrm{Br})$ (0.5 equivalent) was added and the mixture stirred at $50{ }^{\circ} \mathrm{C}$ overnight. The solvent was then evaporated. Purification of the residue by column chromatography (cyclohexane/ethyl acetate, 9:1) gave URB602 as a white solid. URB602 was administered by intracerebroventricular injection. Briefly, the pups were anesthetized and placed in a stereotaxic frame. Intracerebroventricular injections were made into the right lateral ventricle using a $5 \mu \mathrm{l}$ Hamilton syringe. Four microliters containing different doses of URB602 (dissolved in phosphate buffered saline (PBS) containing $52.5 \%$ dimethylsulfoxide) or the corresponding volume of vehicle was injected $30 \mathrm{~min}$ before $\mathrm{HI}$. 


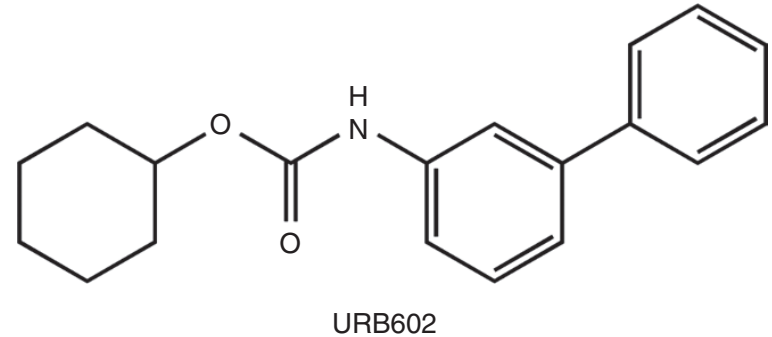

Figure 4. Chemical structure of compound URB602.

\section{Long-Lasting Behavioral Studies}

Each experiment included the following groups $(n=10)$ : (i) vehicletreated control animals (control), (ii) URB602-treated control animals (URB602), (iii) vehicle-treated ischemic animals (HI), and (iv) URB602-treated ischemic animals (HI + URB602).

T-maze. The apparatus was a T-maze with $11-\mathrm{cm}$-wide floor and $18-\mathrm{cm}$-high walls. The stem of both arms was $40 \mathrm{~cm}$ long. Lighting was provided by a lamp suspended above the apparatus. The start arm contained a guillotine door. Spontaneous alternation refers to the tendency of the animal to enter opposite goal boxes in consecutive unrewarded trials in a T-maze. Starting on PN28, each animal was given three trials in the T-maze, giving a chance for two alternations, and the data recorded at $0 \%, 50 \%$, or $100 \%$ alternation for each animal.

Morris maze. Animals were tested starting from PN45. In this task, animals must find a submerged pedestal located below the surface of water (white colored). Animals were evaluated in a training-to-criterion test. The test started by placing the animal in the water facing the wall of the pool in the opposite position of the one containing the pedestal. If the animal located and climbed onto the pedestal, it was permitted $30 \mathrm{~s}$ on the pedestal before the next trial started. If the animal did not find the pedestal within $120 \mathrm{~s}$ it was placed directly on the pedestal and allowed a 30 -s rest period. The performance criterion was three trials in a row with an average time before escape of $<20 \mathrm{~s}$. When the animal reached the criterion, it was transferred to the next location the next day. Each animal received a maximum of 32 trials for each of the three positions of the pedestal. Data are expressed as the number of sessions needed to reach the criterion.

After $15 \mathrm{~d}$ (PN72), each rat was tested for eight additional trials with the pedestal placed in the third position. Each trial lasted $1 \mathrm{~min}$ and the time taken to find the platform was recorded. If the animal located and climbed onto the pedestal within the $1 \mathrm{~min}$ trial, it was permitted to stay $30 \mathrm{~s}$ on the pedestal before the next trial started. If the animal did not find the pedestal within $1 \mathrm{~min}$ it was placed directly on the pedestal and allowed a 30-s rest period. Data of the recall section are expressed as the total time taken to find the pedestal in the eight trials.

\section{Assessment of Brain Injury}

Brain injury was evaluated on PN14 or at the end of the long-term experiments (PN80). Animals were anesthetized, euthanized by decapitation, and the brains immediately frozen in dry ice. To evaluate tissue injury, coronal sections ( $40 \mu \mathrm{m}$ thick) of the brain of each animal were cut on a cryostat and thaw-mounted onto acid-washed subbed slides (gelatin and chrome alum). Sections were then postfixed with $4 \%$ paraformaldehyde in PBS and stained with toluidine blue. A computerized videocamera-based image analysis system (NIH Image software) was used to measure cross-sectional areas from the level of the anterior genu of the corpus callosum to the end of the gyrus dentatus. Measurements, based on the intensity and uniformity of the staining, were performed by an experimenter who was blinded to the conditions of the treatment; these measurements included only intact tissue. Volumes of the whole hemisphere, cerebral cortex, and hippocampus were estimated by summing areas and multiplying by the distance between sections $(400 \mu \mathrm{m})$. The percentage reduction for the whole hemisphere or the selected brain regions was calculated by using the formula: $100 \times(\mathrm{L}-\mathrm{R}) / \mathrm{L}$, where $\mathrm{L}$ is the left-side volume (contralateral uninjured area) and $\mathrm{R}$ is the right-side volume (ipsilateral injured area).

\section{Biochemical and Immunohistochemical Analyses}

These experiments were performed on separated groups of ischemic $(n=10)$ and URB602-treated ischemic (HI + URB602; $n=10)$ animals. Animals were killed for biochemical experiments $24 \mathrm{~h}$ after HI. In additional groups of $\mathrm{HI}(n=5)$ and $\mathrm{HI}+\mathrm{URB} 602$ rats $(n=5)$, propidium iodide (Sigma, Milan, Italy; P4170) dissolved in distilled water was injected at $0.5 \mu \mathrm{g} / \mathrm{rat}$ into the right lateral ventricle in a volume of $0.5 \mu \mathrm{l}$ (7). Twenty minutes after injection, animals were killed and perfusion-fixed with $4 \%$ paraformaldehyde in $0.1 \mathrm{~mol} / \mathrm{l} \mathrm{PBS}$.

Western blot analysis. This analysis was performed on ischemic and contralateral cortices of rats in the HI and HI + URB602 groups. Samples (50 $\mu \mathrm{g}$ protein; Bradford dye-binding procedure, BioRad Laboratories, Segrate, Italy) were separated onto SDS-polyacrylamide gels and probed with a cleaved caspase- 3 antibody (1:1000, polyclonal; Cell Signaling Technology, Danvers, MA). Immunoblots were processed with horseradish peroxidase-conjugated antirabbit antibody (1:4000) and detected using the ECL system (Amersham Pharmacia Biotech, Milan, Italy). A monoclonal antibody against $\beta$-actin (1:4,000, Santa Cruz Biotechnology, Segrate, Italy) was used as control for protein gel loading. Blots were analyzed using the NIH Image software. Data were normalized to those of $\beta$-actin and expressed as optical density integration.

Immunohistochemistry. Pups were deeply anesthetized and perfusionfixed with $4 \%$ paraformaldehyde in $0.1 \mathrm{~mol} / 1 \mathrm{PBS}$. Brains were rapidly removed, placed on ice, and processed for antigen retrieval by immersing overnight in $10 \mathrm{mmol} / \mathrm{l}$ sodium citrate buffer $\left(\mathrm{pH} 6.0,4^{\circ} \mathrm{C}\right)$ and boiling in the same buffer for $3 \mathrm{~min}$. After boiling, brains were cryoprotected with $30 \%$ sucrose/PBS $\left(72 \mathrm{~h}, 4^{\circ} \mathrm{C}\right)$. Brain sections (thickness $20 \mu \mathrm{m}$ ) were incubated with $1.5 \%$ normal blocking serum for $1 \mathrm{~h}$ at room temperature and then overnight at $4{ }^{\circ} \mathrm{C}$ with anticleaved caspase-3 (1:50, polyclonal; Cell Signaling Technology, Danvers, MA). Fluorescein isothiocyanate-conjugate horse antirabbit IgG (1:200, Santa Cruz Biotechnology) was used to demonstrate immunoreactivity of cleaved caspase-3 as green fluorescence. The specificity of the reactions was evaluated in some slices by omitting the primary antibody from the incubation medium.

Cell counting. Cell counting was conducted in three different regions of the hippocampus on $\times 20$ microscopic images using a BX-51 Olympus microscope. Positive cells were counted using the NIH Image software in three separate fields of each region of the hippocampus in slices cut at the level A 3750 of the Koning and Klippel stereotaxic atlas. Five animals in each group were analyzed.

\section{Data Analysis}

The Mann-Whitney $U$ test was used to analyze left and right choices in the T-maze. Data from the training-to-criterion test in the Morris water maze were analyzed using the Kruskal-Wallis test followed by Dunn's multiple comparison test and data from the retention test by one-way ANOVA followed by Newman-Keuls multiple comparison test. Wholebrain or regional volumes after injury were analyzed by Mann-Whitney $\mathrm{U}$ test or by one-way ANOVA followed by Newman-Keuls post hoc test. The relative intensities of the bands detected by western blot were analyzed using NIH Image 1.62 software and statistical analyses performed by one-way ANOVA; data are presented as mean \pm SEM.

\section{ACKNOWLEDGMENTS}

We thank Claudia Scopa for the excellent technical assistance and Kevin Worrell for his helpful hints.

\section{STATEMENT OF FINANCIAL SUPPORT}

This work was supported by Italian MiUR (Ministero dell'Università e della Ricerca) and University of Urbino "Carlo Bo."

\section{REFERENCES}

1. Piomelli D. The molecular logic of endocannabinoid signalling. Nat Rev Neurosci 2003;4:873-84.

2. Galve-Roperh I, Aguado T, Palazuelos J, Guzmán M. Mechanisms of control of neuron survival by the endocannabinoid system. Curr Pharm Des 2008; 14:2279-88.

3. Klein TW, Lane B, Newton CA, Friedman H. The cannabinoid system and cytokine network. Proc Soc Exp Biol Med 2000;225:1-8. 
4. Mechoulam R, Panikashvili D, Shohami E. Cannabinoids and brain injury: therapeutic implications. Trends Mol Med 2002;8:58-61.

5. Muthian S, Rademacher DJ, Roelke CT, Gross GJ, Hillard CJ. Anandamide content is increased and CB1 cannabinoid receptor blockade is protective during transient, focal cerebral ischemia. Neuroscience 2004;129:743-50.

6. Degn M, Lambertsen KL, Petersen G, et al. Changes in brain levels of $\mathrm{N}$-acylethanolamines and 2-arachidonoylglycerol in focal cerebral ischemia in mice. J Neurochem 2007;103:1907-16.

7. Fernández-López D, Martínez-Orgado J, Nuñez E, et al. Characterization of the neuroprotective effect of the cannabinoid agonist WIN-55212 in an in vitro model of hypoxic-ischemic brain damage in newborn rats. Pediatr Res 2006;60:169-73.

8. Zhang M, Martin BR, Adler MW, Razdan RK, Ganea D, Tuma RF. Modulation of the balance between cannabinoid $\mathrm{CB}(1)$ and $\mathrm{CB}(2)$ receptor activation during cerebral ischemic/reperfusion injury. Neuroscience 2008;152:753-60.

9. Pellegrini-Giampietro DE, Mannaioni G, Bagetta G. Post-ischemic brain damage: the endocannabinoid system in the mechanisms of neuronal death. FEBS J 2009;276:2-12.

10. Zhang M, Martin BR, Adler MW, et al. Modulation of cannabinoid receptor activation as a neuroprotective strategy for EAE and stroke. J Neuroimmune Pharmacol 2009;4:249-59.

11. Panikashvili D, Simeonidou C, Ben-Shabat S, et al. An endogenous cannabinoid (2-AG) is neuroprotective after brain injury. Nature 2001; 413:527-31.

12. Shen M, Thayer SA. Cannabinoid receptor agonists protect cultured rat hippocampal neurons from excitotoxicity. Mol Pharmacol 1998;54:459-62.

13. van der Stelt M, Veldhuis WB, Bär PR, Veldink GA, Vliegenthart JF, Nicolay K. Neuroprotection by Delta9-tetrahydrocannabinol, the main active compound in marijuana, against ouabain-induced in vivo excitotoxicity. J Neurosci 2001;21:6475-9.

14. Fernández-López D, Pazos MR, Tolón RM, et al. The cannabinoid agonist WIN55212 reduces brain damage in an in vivo model of hypoxic-ischemic encephalopathy in newborn rats. Pediatr Res 2007;62:255-60.

15. Alonso-Alconada D, Alvarez FJ, Alvarez A, et al. The cannabinoid receptor agonist WIN 55,212-2 reduces the initial cerebral damage after hypoxicischemic injury in fetal lambs. Brain Res 2010;1362:150-9.

16. Gaetani S, Dipasquale P, Romano A, et al. The endocannabinoid system as a target for novel anxiolytic and antidepressant drugs. Int Rev Neurobiol 2009;85:57-72.

17. Landucci E, Scartabelli T, Gerace E, Moroni F, Pellegrini-Giampietro DE. $\mathrm{CB} 1$ receptors and post-ischemic brain damage: studies on the toxic and neuroprotective effects of cannabinoids in rat organotypic hippocampal slices. Neuropharmacology 2011;60:674-82.

18. Carloni S, Carnevali A, Cimino M, Balduini W. Extended role of necrotic cell death after hypoxia-ischemia-induced neurodegeneration in the neonatal rat. Neurobiol Dis 2007;27:354-61.

19. Balduini W, De Angelis V, Mazzoni E, Cimino M. Long-lasting behavioral alterations following a hypoxic/ischemic brain injury in neonatal rats. Brain Res 2000;859:318-25.

20. Carloni S, Perrone S, Buonocore G, Longini M, Proietti F, Balduini W. Melatonin protects from the long-term consequences of a neonatal hypoxic-ischemic brain injury in rats. J Pineal Res 2008;44:157-64.

21. Balduini W, De Angelis V, Mazzoni E, Cimino M. Simvastatin protects against long-lasting behavioral and morphological consequences of neonatal hypoxic/ischemic brain injury. Stroke 2001;32:2185-91.

22. Schomacher M, Müller HD, Sommer C, Schwab S, Schäbitz WR. Endocannabinoids mediate neuroprotection after transient focal cerebral ischemia. Brain Res 2008;1240:213-20.

23. Dinh TP, Kathuria S, Piomelli D. RNA interference suggests a primary role for monoacylglycerol lipase in the degradation of the endocannabinoid 2-arachidonoylglycerol. Mol Pharmacol 2004;66:1260-4.

24. Makara JK, Mor M, Fegley D, et al. Selective inhibition of 2-AG hydrolysis enhances endocannabinoid signaling in hippocampus. Nat Neurosci 2005;8:1139-41.

25. Hohmann AG, Suplita RL, Bolton NM, et al. An endocannabinoid mechanism for stress-induced analgesia. Nature 2005;435:1108-12.

26. King AR, Duranti A, Tontini A, et al. URB602 inhibits monoacylglycerol lipase and selectively blocks 2-arachidonoylglycerol degradation in intact brain slices. Chem Biol 2007;14:1357-65.

27. Comelli F, Giagnoni G, Bettoni I, Colleoni M, Costa B. The inhibition of monoacylglycerol lipase by URB602 showed an anti-inflammatory and anti-nociceptive effect in a murine model of acute inflammation. Br J Pharmacol 2007;152:787-94.

28. Panikashvili D, Shein NA, Mechoulam R, et al. The endocannabinoid 2-AG protects the blood-brain barrier after closed head injury and inhibits mRNA expression of proinflammatory cytokines. Neurobiol Dis 2006;22:257-64.

29. Zhang J, Chen C. Endocannabinoid 2-arachidonoylglycerol protects neurons by limiting COX-2 elevation. J Biol Chem 2008;283:22601-11.

30. Rice JE 3rd, Vannucci RC, Brierley JB. The influence of immaturity on hypoxic-ischemic brain damage in the rat. Ann Neurol 1981;9:131-41.

31. Tarzia G, Duranti A, Tontini A, et al. Design, synthesis, and structureactivity relationships of alkylcarbamic acid aryl esters, a new class of fatty acid amide hydrolase inhibitors. J Med Chem 2003;46:2352-60. 